\title{
A calculus student's understanding of graphical approach to the derivative through quantitative reasoning
}

\author{
Aytug Ozaltun-Celik \\ Pamukkale University,Turkey
}

The concept of derivative is used in many areas including applied problems and requiring mathematical modelling in different disciplines. One of the most important approaches for teaching the derivative is to support students in visualizing the concept. Also, it is necessary to shift researchers and teachers' focuses to students' dynamic mental actions while learning derivative in order to conduct effective teaching process. With this necessity, I focused on the perspective of quantitative reasoning related to the graphical approach to the derivative. This study aims to reveal a calculus student's mental actions related to the graphical approach to the derivative. The data were collected from a first-year calculus student engaged in the task requiring graphical interpretation of the derivative. Results showed that the student's understanding of the slope shaped her inferences about the tangent line because the quantity of ratio is prior knowledge for learning the instantaneous rate of change. Besides, as the student had the idea of correspondence related to the concept of function, she had difficulties in interpreting the global view of the derivate. This result suggests that having global view of the derivative requires a strong understanding of function and rate.

Keywords: Calculus student, derivative, graphical approach, quantitative reasoning

\section{Introduction}

The derivative of a function is a fundamental concept for the basis of calculus (García et al., 2011) and is used in many areas including requiring mathematical modeling of several situations in different disciplines such as engineering, physics, economics, etc. This concept was historically constructed as a way to represent rate of change which explains how one quantity changes in relation to another quantity (Weber et al., 2012). Thus, understanding the derivative requires a wide intuitive base of examples and related perceptions, especially concerning the concept of the rate of change in real-life problems (Weigand, 2014). Thompson (1990) has emphasized the idea that a rate is conceived of as constituting a functional relationship may be a foundation for the derivative in calculus because it is consistent with conceptions of a single-variable derivative evaluated at a point (i.e., an instantaneous rate of change) as being the slope of a tangent line. Especially, visualizing a graph supports students to construct a 
tangent as an instantaneous rate of change by zooming in on a sufficiently small portion of the curve (Tall, 2010). Tall (1997) has related to the graphical representation of the derivative with the qualitative actions of visualizing and conceptualizing. Students might thus transfer their meanings to the instantaneous rate of change which is an important cognitive action for learning derivative and engage in sense making of the derivative beyond applying the derivative formulas (Samuels, 2017). The slopes of secant line and tangent line are quantities which have to be measured necessarily in quantification process of derivative graphically. Students first think the slope of secant line and then use the values of the slopes for different secant lines to get the slope of the tangent line at a point. The relationship between the derivative of a function at a point and the slope of the tangent line at that point forms a foundation for understanding the derivative as function (Asiala et al., 1997). As they progress, students can visualize the quantities on the graph and imagine the slope of all tangent lines at any points on the curve. Considering that many calculus students have difficulties in visualizing of the rate of change of two quantities (Hausknecht \& Kowalczyk, 2008), the graphical representation of derivative becomes important for visualization and learning. Additionally, students need to progress developmentally based on graphical tasks about the derivative in order to interpret it graphically. Because many students interpret a graphical problem about the derivative algebraically, when a curve and its tangent line are given (Asiala et al., 1997). However, it would require that students be able to meaningfully connect the ideas of slope (instantaneous rate of change) in both algebraic ratio and geometric ratio for perceiving as an internal concept (Nagle et al., 2019). Based on these ideas, I focused on the graphical approach to the concept of derivative and the quantities related to the graph. On hand, this study proposes a task implementation for supporting students' mental actions regarding instantaneous rate of change, on the other hand, presents reveal a calculus student's mental actions related to the graphical approach to the concept of derivative.

Most studies on graphical representation of the derivative have been based on using a dynamic mathematic software (i.e. Borji et al., 2018a; Delos Santos \& Thomas, 2005). Since high school teachers or college instructors may not have any opportunity to use technological tools in terms of facilities or technological knowledge, a learning task challenging student to imagine the dynamic process of the derivative without using any software is of importance. The task in this study can provide teachers to teach the derivative dynamically without using any mathematics or geometry 
software. Additionally, presenting a typical calculus student's mental actions while engaging in a such task may give readers opportunities to revise and use this kind of task while teaching the derivative. Since the derivative is constructed by human pattern-finding tendencies, rather than being self-existent (Jones \& Watson, 2018), the results from a calculus student's mental actions may help mathematics educators to understand students' developmental progression related to the derivative and to design different teaching processes to support in making connections among different representations. Thus, I seek to respond this research question: "What are a calculus student's mental actions related to the graphical interpretation of the derivative by quantitative reasoning?”

\subsection{Literature Review: The Concept of Derivative}

There are several studies on students' understanding of the derivative (Asiala et al., 1997; Borji et al., 2018a; Borji et al., 2018b; Delos Santos \& Thomas, 2005; Firouzian, 2013; García et al., 2011; Habre \& Abboud, 2006; Jones \& Watson, 2018; Kendal \& Stacey; 2000; Kertil, 2014; Park et al., 2013; Serhan, 2000; Verhoef et al., 2015; Zandieh, 2000). Some researchers (Asiala et al., 1997; Zandieh, 2000) explained the developmental progressions during learning the concept of derivative while some (i.e. Delos Santos \& Thomas, 2005; Habre \& Abboud, 2006; Verhoef et al., 2015) studied on the teaching process of the derivative. Asiala et al. (1997) who examined calculus students' graphical understanding of the derivative suggested the graphical paths and coordinated these paths with the analytic paths (p.10):

1. The action of connecting two points on a curve to form a chord which is a portion of the secant line through two points together with the action of computing the slope of the secant line through the two points.

2. Interiorization of the actions in point to a single process as the two points on the graph get closer and closer together.

3. Produce the tangent line as the limiting position of the secant lines and also produce the slope of the tangent line at a point on the graph of a function.

Similarly, Zandieh (2000) has developed a framework for exploring students' understanding of derivative. For four contexts (graphical, verbal, paradigmatic physical, symbolic), Zandieh has elaborated three layers (ratio-limit-function) during learning process and stated that these layers could be seen as dynamic process and as static objects which are linked in a chain: 
The ratio process takes two objects (two differences, two lengths, a distance and a time, etc.) and acts by division. The reified object (the ratio, slope, velocity, etc.) is used by the next process that of taking a limit. The limiting process 'passes through' infinitely many of the ratios approaching a particular value (the limiting value, the slope at a point on a curve, instantaneous velocity). The reified object, the limit, is used to define each value of the derivative function. The derivative function acts as a process of passing through (possibly) infinitely many input values and for each determining an output value given by the limit of the difference quotient at that point. The derivative function may also be viewed as a reified object just as any function may. (p.107)

While students are studying on the graphical context at the ratio layer, they find the slope of a secant line through two points on the graph. Secondly, they use the concept of limit to find the slope of the tangent line at a point by thinking of approximation points on the curve to a specific point. Finally, comprehending the derivative as a function requires understanding that the slope is different for different values of the independent variable. Borji et al. (2018b), one of the studies analyzing students' mental actions about the derivative, examined fourteen university students' understanding on the graph of the function and its derivative by using the perspectives APOS (Action, Process, Object, Schema) and OSA (Onto-Semiotic Approach) which they saw them complementary. Their results showed that ten students were at the intra level which is the lowest level of development of the Schema. These students at that level could perform some mental constructions of the genetic compositions considered as actions, but they did not consider some of the propositions of the epistemic configuration and could not sketch the graph of the derivative function. Nagle et al. (2019) proposing a framework for slope using APOS expressed that, for the construction of a derivative as a function $f^{\prime}$, where at each point on the graph of $f$, a student has the dynamic imagery of secant lines approaching the tangent line at the point (requiring a Process of slope) and of tangent lines moving along the graph of $f$ with the slopes corresponding to the values of $f^{\prime}$ (resulting from Actions on a Process of slope). These frameworks provide important insights us how students have mental actions in the learning process of the derivative.

Additionally, there are considerable evidence that the calculus and high school advanced algebra students have many difficulties about the derivative (e.g. Firouzian, 2013; Kendal \& Stacey, 2000; Sánchez-Matamoros et al., 2015). Firouzian (2013) found that most calculus students did not explain the derivative with the idea of slope of the tangent line and instantaneous rate of change. Sánchez-Matamoros et al. (2015) assert that students cannot understand the relation between the limit and the derivative and also relation between derivative of a function at a point and global view 
of derivative. Kendal and Stacey (2000) stated that only the most capable students were successful at numerical, graphical and symbolic representations of the derivative. These challenges bring to light the necessity of effective teaching process prompting students to have deeper meanings of the derivative.

Some of these researchers who focused on the teaching process of the derivative have offered using the tasks supported by technological tools (Borji et al., 2018a; Delos Santos \& Thomas, 2005; Habre \& Abboud, 2006; Kendal \& Stacey, 2000; Verhoef et al., 2015) while some have asserted that mathematical modelling activities have helped students to have conceptual learning of the derivative (Kertil, 2014; Park et al., 2013). Delos Santos and Thomas (2005) conducted a case study with two students and they examined the effectiveness of a module using graphical calculators on students' understanding of the derivative concept. They have observed that the students move from a procedural perspective to a more concept-oriented view of derivative through this module. Habre and Abboud (2006) focused on ten undergraduate students' understanding of the function and its derivative during a reformed Calculus-I including graphing calculators and a dynamical calculus software program. After all courses, some students explained the derivative as being the slope of the tangent line at a point and some of them explained it in terms of the instantaneous rate of change. Similarly, Borji et al. (2018a) used Maple software to improve university students' graphical understanding of derivative. They conducted an experimental study in which they designed three activities based on APOS-ACE (Action, Process, Object, Schema-Activities in class, Classroom discussion, Exercises) framework and emphasized interiorization of the process of calculating the slope of a secant line drawn by connecting two points on a curve. The results showed that the students in the experimental group had a deeper understanding of derivative. These researches acknowledge that the dynamically learning environments involving process meaning of the derivative might support students' mental actions. The results from the previous researches present students' understanding of derivative after they completed the learning process. In this study, I alternatively focus on the student's quantitative reasoning while engaging in a new task for her. Since the student's mental actions of the derivative shaped while studying on the task, this study gives significant approaches related to learning processes of the derivative to the mathematics teachers to help their students to have conceptual understanding or to the researchers to conduct larger teaching experiments. As a different aspect from the above studies, this study draws on the quantitative reasoning which can serve as the conceptual root stalk 
for many different approaches to algebra and calculus and make sense the relations among the quantities (Smith III \& Thompson, 2007).

\subsection{Theoretical Perspective}

In this section, I outline the perspective of quantitative reasoning that guided my graphical approach to the derivative and then explain how I use this perspective.

Quantitative reasoning is the analysis of a situation into a quantitative structurea network of quantities and quantitative relationships (Thompson, 1990, p.12). It involves an individual's mental actions when conceiving of a situation, constructing measurable attributes of the situation and constructing about relationships between conceived quantities (Moore, 2013). In this perspective, since relations among quantities are important, quantitative reasoning is closely related to the kind of reasoning customarily emphasized in algebra instruction (Thompson, 1993). The Common Core State Standards for Mathematics (CCSSI, 2010) emphasizes the importance of students' quantitative reasoning, which is defined as making sense of the quantities in the problem situations and the relations between these quantities, in other words, as the mental actions necessary for students to learn mathematics. Accordingly, quantitative reasoning can be considered as an aim of teaching, and mental operations and conceptual structures that enable quantitative reasoning should be elaborated (Thompson, 1990).

Thompson (1990) elaborated the quantitative reasoning by defining several concepts such as quantity, quantification, quantitative operations, value. A quantity is a quality of something that one has conceived as admitting some measurement process (Thompson, 1990). In this study, the horizontal distance (purple line segment in the Figure 1) and the vertical distance (orange line segment) between two points which the secant line (red line) intersects on the curve are quantities. By comparing these quantities multiplicatively, the slope of the secant line (average rate of change $[(f(x+\Delta x)-f(x)) / \Delta x]$ or steepness) are produced. Comparing quantities multiplicatively is quantitative operation which is the conception of two quantities being taken to produce a new quantity (Thompson, 1990). 


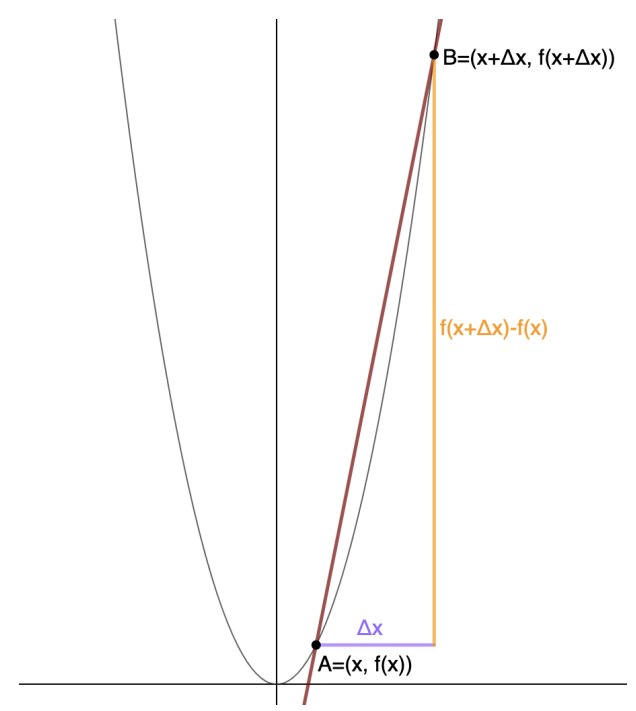

Figure 1. Secant line on the curve $f(x)=x^{2}$

The process of measuring the value of the slope of secant line on the curve is quantification. This measurement process is precursor step in constructing the slope of tangent line (instantaneous rate of change) which is another quantity. In this process, by moving a point through curve towards the fixed point, a new secant line which its steepness (slope or rate of change) is changing is formed. As the point A approximates to the point $\mathrm{B}$ in the Figure 2, since changing of the change in $\mathrm{x}$-axis is less than changing of the change in y-axis based on the quadratic function, steepness is increasing, and a new secant line is formed. If students are given the opportunity of forming the secant lines based on this idea, their emergent shape thinking which "involves understanding a graph simultaneously as what is made (a trace) and how it is made (covariation)"(Moore \& Thompson, 2015, p. 785) which is may be improved. Secant lines and tangent line are constructed based on emergent This movement also leads to think a rotating secant line. The activity of rotating this secant line around the fixed point on which tangent line would be drawn or moving the point A towards the point B through curve prompts to reason about the idea of approximating (Figure 2). 


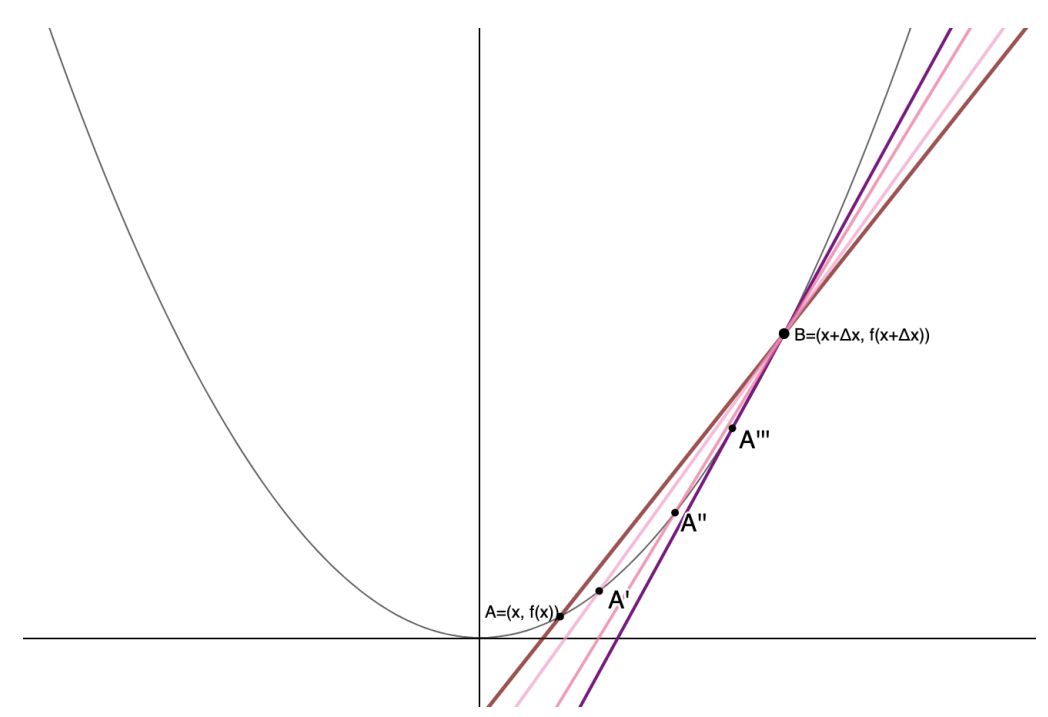

Figure 2 . The activity of moving the point A to the point B

These activities are important in constructing the slope of the tangent line at the point B (new quantity). Based on these activities, the tangent line and its slope cannot visible but imaginable. The instantaneous rate of change is the result of an approximation producing average rate of change over smaller and smaller intervals. Since this approximation process is related to the concept of limit, quantifying the instantaneous rate of change can be by calculating the limit of average rate of change:

$$
\lim _{\Delta x \rightarrow 0} \frac{f(x+\Delta x)-f(x)}{\Delta x}
$$

After students construct the slope of tangent line at a point, they think that slopes of different tangent lines on the curve change and notice derivative as a function. These actions based on the perspective of quantitative reasoning are related to the ideas from Zandieh's (2000) and Asiala et al's (1997) frameworks. However, since quantitative reasoning as a way of defining the mental actions of a student who understands a situation, constitutes quantitative situations, associates, organizes and uses these quantities to make the problem situation meaningful (Weber et al., 2014), it helps us to understand students' dynamic mental actions which support their conceptual learning by allowing them to make connections between different concepts and evaluate situations within quantitative structures. 


\section{Methodology}

The methodology for this study was a qualitative case study having the aim of revealing a calculus student's graphical understanding of the derivative. The case was the calculus student's mental actions based on the quantitative reasoning. The participant, pseudonym named Amelia (female), was a typical first-year calculus student and was willingness to participate to the study. I carried out a task-based interview with Amelie to understand her mathematics. Goldin (2000) emphasized the characteristics of task-based interviews different from conventional interviews:

...task-based interviews make it possible to focus research attention more directly on the subjects' processes of addressing mathematical tasks, rather than just on the patterns of correct and incorrect answers in the results they produce. Thus, there is the possibility of delving into a variety of important topics more deeply than is possible by other experimental means topics such as complex cognitions associated with learning mathematics, mechanisms of mathematical exploration and problem solving, relationships between problem solving and learning, relationships between affect and cognition, and so forth. (p. 520)

In the study, the task-based interview helped me to understand Amelie's complex mental actions related to the derivative. The task was presented to Amelia out of the class by a paper before she did not engage in a learning process of derivative as a part of the calculus course. I conducted the interview in one session lasting about two and a half hours. While she was engaging in the task, I continually asked the underlying reasons of her thoughts and I videotaped all process.

\subsection{The Task and Data Collection}

The focus of the task is on the graphical interpretation of the derivative. The steps of the task are as follows (Figure 3): 
Step 1: How do you find slope of a line? Explain.

Step 2: Interpret this function, $f: \mathbb{R} \rightarrow \mathbb{R}, f(x)=x^{2}$ ? What can you say about it? Explain.

Step 3: Sketch the graph of this function.

Step 4: Determine a point $A$ on the graph and draw a tangent line at a point $A$.

Step 5: Find the slope of this tangent line. Explain your steps.

Prompt questions:

- What information is given about the line?

- What do you know about this line?

(If the student has not a logical idea for finding the slope, continue with Step 6)

Step 6: Assume that there is a ring $(P)$ on the graph. Is it possible to remove the ring through the curve? What do you think?

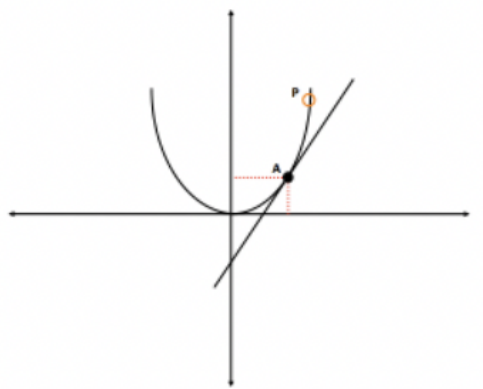

Prompt questions:

- What do you think about removing the ring for finding slope of the tangent line?

- What can you say about the function considering this movable action?

- What does moving of the ring work for finding the slope of the tangent line?

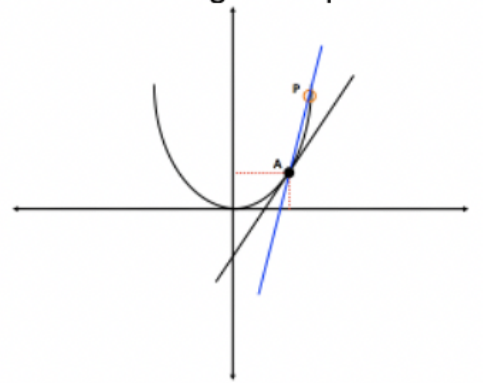

(If the student has not a logical idea for finding the slope, continue with Step 7.)

Step 7: Examine the changes in the secant line. (These drawings are given to the student statically.)
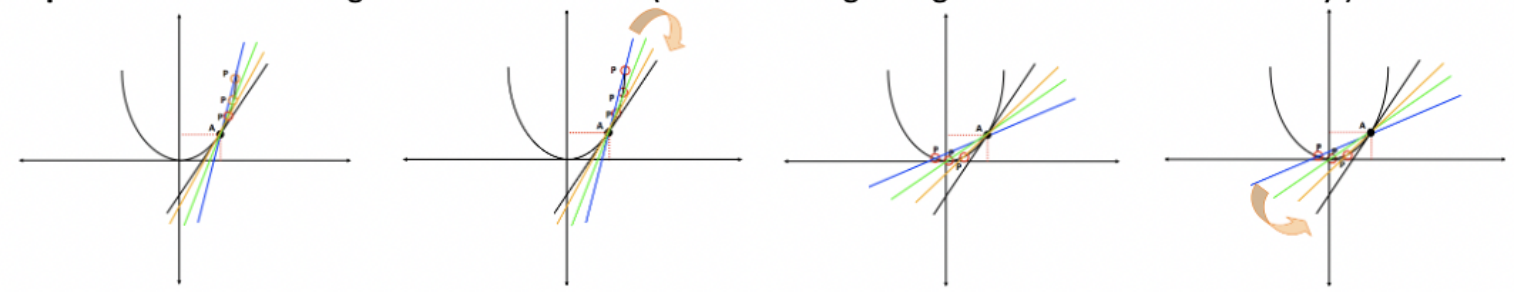

Step 8: Which steps do you follow for finding the slope of tangent line at point of $(1,1)$ ?

Step 9: Find the slope of tangent line at the different points (for $x=0, x=2, x=-1$ et al.) on the graph and draw a table representing matching values of slope to values of $\mathrm{x}$-axis of points.

Prompt questions:

- What does the matching point out?

- Can you find the slope of the tangent line at all points on the graph depending on their values of $\mathbf{x}$ ? Explain.

- Is there a value of slope corresponding to every $x$-value?

- What does this relation mean?

Step 10: Construct the function representing the value of slope of tangent line at any point depending on the value of $x$-axes of that point. 
Step 1-4. In the first step of the task, students are expected to think the slope of a line. By this step, students need to activate their knowledge about the slope as a constant rate of change. In the further steps they might build new ideas on their knowledge regarding the quantity of slope. In the task, the function is a quadratic function because most calculus students know the curve representing its graph. The aim of second step is to reveal students' meanings related to the quadratic function. For instance, they may have a misunderstanding that any segment drawn through two points on the parabola is linear and such an understanding may be a block for their reasoning about the slope of secant line and tangent line. The third step requires students to draw the graph of the function. The factors such as drawing graph, determining a point on the graph should not lead to some obstacles so that they can reason about the slope of a tangent line and derivative. Students are asked to draw a tangent line on it after they draw the graph.

Step 5-7. In the $5^{\text {th }}$ step, it is assumed that students might have difficulties about reasoning about the slope of a tangent line because they can calculate the slope of a line by using two points on the line. However, they only have limited information about the tangent line. They may easily determine the point on the curve on which the tangent line is drawn. But this point is not identified at this stage. For this reason, teachers can ask prompt questions to them. These questions may trigger students to think the tangent line as a line and to notice that they need two points at least to interpret the slope of the tangent line. Then, they continue to work on the task by assuming that there is a ring on the curve and that they can move the ring on the curve. In this study, the images given to students are static. In different teaching processes, they may be asked to work in a dynamic technological environment such as GeoGebra. The ring on the curve is representing another point and students thus might form a secant line through the ring and the point on which the tangent line is drawn. Also, since the ring can move through all points of the curve, students may notice continuity for the function. In other words, the idea of moving a ring through the curve helps students to make inferences that the function is continuous. Since being continuous is necessity for differentiable, using this imaginable activity is of importance. As the ring moves, the changes in the x-axis and in the y-axis change differently because of the quadratic function and thus the slope of the secant line changes. When students interpret rate of change of the secant lines before drawing the lines, they construct secant lines based on emerging shape thinking. This thinking supports them in using the slope of secant lines to get the slope of the tangent line. Students need to make 
inferences about the secant lines in this step but if not, in the 7th step they examined the graphs given. It is assumed that this action might support students' learning for the relation between average rate of change and instantaneous rate of change. In this step, several prompt questions may be asked to students. These steps are important for the students to realize quantitative operations significant ideas for the slope of tangent line.

Step 8. In this step, students work on quantification process for a slope of the tangent line at a specific point $(1,1)$. They determine another point on the curve correspondence to the ring and gradually they approximate it to the $(1,1)$ on the curve. They need to think on the points which their $\mathrm{x}$-values are smaller than 1 (as the $\mathrm{x}$ value of the specific point of $(1,1))$.

Step 9-10. After interpreting the slope of a tangent line at a specific point on the curve by the idea of instantaneous rate of change, students are asked to think the slope of tangent line at different points. They are expected to match $\mathrm{x}$-values of the points to the slope of tangent lines drawn on that points. Thus, it is assumed that they might notice a new function and have a global view of the derivative.

\subsection{Data Analysis}

The data analysis process consisted of ongoing analysis simultaneous to the data collection process and retrospective analysis after the interview. By the ongoing analyses, I focused on the student's activities and quantitative operations. This process supported me an insight about the student's thoughts and was precursor for the retrospective analysis.

After interview, I did retrospective analysis. The retrospective analysis method provided me to examine how the student has thought and to understand what actions have shaped her thoughts (Battista \& Clement, 2000). For retrospective analysis, I first transcribed the video camera recordings verbatim and examined the student's gestures and talks based on the perspective of quantitative reasoning. I divided the transcription into the parts accordance with the steps of the task. I first coded the student's talks about the slope in terms of the rate of change. In this stage, I focused on her talks by considering the idea of the multiplicative comparison which is an important operation for the slope. Then, I continued the analysis process of her ideas about the slope of a tangent line in a descriptive way. For next steps of the task, I focused on the student's quantitative operations, quantitative relations and the quantities and I coded her talks by continuous comparative analysis. I initially 
examined her quantitative operations and then, the quantities and concepts she constructed by these quantitative operations. By this coding, I aimed to reveal the quantities she produced during the interview. Based on the coding process, I modelled the student's mental actions in the process of quantification.

\section{Results}

In the section of results, I first present the student's knowledge about the slope of a line. Then, I illustrate her initial understanding of the slope of a tangent line at a point on the curve. Lastly, I provide evidence on the student's quantification and quantitative reasoning about graphical approach to derivative.

\subsection{Amelie's Understanding of Slope of a Line}

In the task, I initially asked the student's knowledge about the slope of a line. By this question, I also aimed to reveal whether the student had an understanding of slope related to the idea of rate of change. She explained that she could find the slope with the ratio of $y$-intercept value over $\mathrm{x}$-intercept value. Based on this explanation, I thought that she did not think the amounts of change for slope and only considered intercept points of line with the coordinate system. In order to elaborate Amelie's thinking, I asked her to explain ideas further. She drew a coordinate system and indicated what she meant on it [Excerpt 1].

Excerpt 1.

Amelie: This is a line. I determine two points on this line and identify one of them as $(\mathrm{x} 1, \mathrm{y} 1)$, the other one as $(\mathrm{x} 2, \mathrm{y} 2)$. In order to find the slope of this line, I calculate the value of $\mathrm{x} 2-\mathrm{x} 1$, then the value of $\mathrm{y} 2-\mathrm{y} 1$. The ratio of $\mathrm{y} 2-\mathrm{y} 1$ over $\mathrm{x} 2-\mathrm{x} 1$ would be equal to the slope of the line.

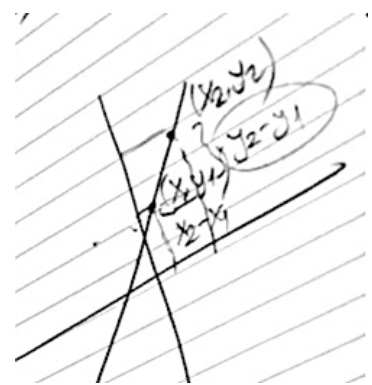

Figure 4. Amelie's diagram representing the slope 
[She writes the equation $\left.\frac{y_{2}-y_{1}}{x_{2}-x_{1}}=m\right]$

Amelie mentioned the ratio of amounts of change in $\mathrm{x}$ and $\mathrm{y}$ values and she wrote the equation for this ratio $\left[\frac{y_{2}-y_{1}}{x_{2}-x_{1}}=m\right]$. However, she explained the slope of the line statically and only considered two points she determined on the line. Also, she did not make any explanation about anything referring that slope could be defined as the amount of change in $y$-axis for every one-unit change in $\mathrm{x}$-axis.

\subsection{Amelie"s Initial Understanding about Slope of Tangent Line}

Amelie easily drew the graph of the function $\left[f(x)=x^{2}\right]$ in the third step and a tangent line at a point on the parabola in the fourth step.

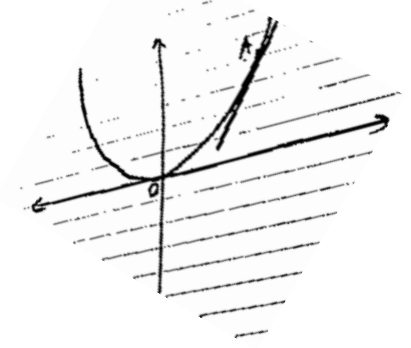

Figure 5. Amelie's diagram for the tangent line

Initially, while she was finding the slope of the tangent line on the parabola, she needed to write algebraically the equation of this tangent line or to determine two points on the tangent line. Since one of these points [A] was also on the curve, she had to identify another point. "...If I know two points on the line, I can draw it and find its slope. I know one point on the line [indicating A] but I cannot find the other one." Just as I hypothetically thought that Amelie might have difficulties for this process, Amelie did not proceed to next step for reasoning the slope. When I pushed her to think of given information about the line, she was still attempted to find the second point on the x-axis. Because of her confusion, Amelie thought the angle formed by the intersection of the line and the x-axis. Her aim was to use the value of the angle for the tangent. I can say that her approach was not paving way towards finding the slope also, because there was not enough information about the angle and the line. She thus could not reason about calculating the value of the slope since there was inadequate information about all ways in finding the slope of tangent line. Her fruitless attempts 
were helping her to feel the existence of alternative ways in finding the slope of the tangent line. This task in the step was thus so valuable.

\subsection{Amelie's Mental Actions During Teaching Process}

I can say that the step in which reasoning the slope of tangent line is engaged is 6th step. In this step, the first aim was to encourage the student to have an understanding about the idea of continuity of the function with the idea of the ring. Continuity of function is a necessary characteristic for the derivative but not enough. For this function, since the ring can be moved through the curve, the function has a derivative at every point in its domain. When I asked Amelie to assume that there was a ring on the graph, she mentioned continuity of the function in its domain and existence of moveableness for the ring through the all curve. Moving the ring through the curve would support the student in imagining the secant lines dynamically.

She then continued the task with Step 7. In this step, while she was examining the first graph, she determined a point correspondence to the ring on the first secant line. Thus, this step challenged her to engage in the quantification process for only one secant line. The slope of the first secant line she calculated was a quantity. By examining the graphs given on the task step by step, she noticed that the rate of change for the secant line gradually would become like to the rate of change for the tangent line at the point A [Excerpt 2].

Excerpt 2.

Amelie: This is point B [the first B point on the blue line], this is point B [indicating the point on the green line], then this is $\mathrm{B}$ [indicating the point on the orange line], and then this is point $B$ [indicating the point on the black secant line]. When the ring is at this point [indicating the point $\mathrm{A}$ ], the slope of the secant line is equal to the slope of the tangent line!

Amelie who had examined the changing graphs reasoned on the situation by considering the points on the secant lines. She qualitatively approximated the point B on the curve to the point $\mathrm{A}$. The task prompted her to make inference about the relation between the secant line and tangent line by the means of moving the ring and rotating the secant line as to being look like the tangent line. Based on these activities, she interpreted that the point B gradually would become to be the point A.

She focused rotating the first secant line around the point A after idea of approximating and imagined as if a new secant line was formed at every stage. Since the point A was not identified in this step, she did not assign a value to slope of the 
any secant line. As it can be understood in the Excerpt 2, she interpreted the changing situation and its result. Amelie's interpretation indicated that she noticed that the slope of the secant line would gradually equal to the slope of the tangent line. The action of rotating prompted her to think of the slope as a steepness.

In the next step, she was asked to draw the tangent line at the point $\mathrm{A}(1,1)$ on the parabola and to find its slope. By depending on the previous step, she first determined the point as $(2,4)$ and draw a secant line through $(1,1)$ and $(2,4)$. Then she calculated the value of slope of this secant line as an average rate of change.

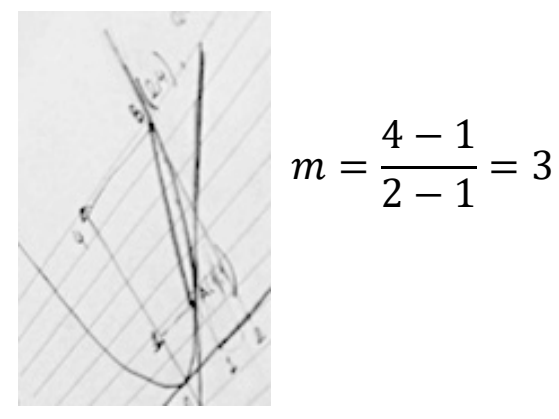

Figure 6. Amelie's diagram related to the slope of the secant line

She determined different points gradually approaching to 1 in way that their $\mathrm{x}$ values were bigger than 1 . These points were $(3 / 2,9 / 4),(5 / 4,25 / 16),(1.1,1.21)$ and (1.01, 1.0201). She drew the secant lines passing through $(1,1)$ and one point which she determined. She then found the values of the slopes of these lines via a calculator and created a table including the values of the slopes and the points (Table 1).

Table 1. Amelie's table for the changing slope values of the secant lines

\begin{tabular}{lc}
\hline Point & Slope \\
\hline $\mathrm{B}(2,4)$ & $m_{B A}=3$ \\
$\mathrm{C}(3 / 2,9 / 4)$ & $m_{C A}=2.5$ \\
$\mathrm{D}(5 / 4,25 / 16)$ & $m_{D A}=2.25$ \\
$\mathrm{E}(1.1,1.21)$ & $m_{E A}=2.1$ \\
$\mathrm{~F}(1.01,1.0201)$ & $m_{F A}=2.01$ \\
\hline
\end{tabular}

She then determined the points gradually approaching to 1 which their x-values were smaller than 1. Similarly, she drew the secant lines by using these points and created a table by computing the slopes of the secant lines by using a calculator (Table 2). This process involved numerical examinations based on her ideas from the previous step as a quantifying process of the slopes (as rate of change). 
Table 2. Amelie's table for the changing values of slopes

\begin{tabular}{lc}
\hline Point & Slope \\
\hline $\mathrm{G}(0.5,0.25)$ & $m_{G A}=1.5$ \\
$\mathrm{H}(0.9,0.81)$ & $m_{H A}=1.9$ \\
$\mathrm{I}(0.99,0.9801)$ & $m_{I A}=1.99$ \\
\hline
\end{tabular}

While the points were approximating to the point A through the curve, she first focused on $\mathrm{x}$-values of the points and then calculated the $\mathrm{y}$-values depending on $\mathrm{x}$ values. Based on this quantifying process, Amelie concluded that slope of the tangent line at $(1,1)$ would be 2 . Determining the points by approximating $(1,1)$ and calculating the values of the slopes of the secant lines supported the student in making inference about the tangent line and its slope.

Excerpt 3.

\begin{abstract}
Researcher: Why did you examine the slopes of the secant lines through the points which their $\mathrm{x}$-values were smaller than 1 ?

Amelie: If the values of the slopes [indicating the lines through latter points] approximated to 3 and these values of slopes [indicating the lines through former points] approximated to 2, the tangent line would not have been the same. This function would be a piecewise function. Because this function is continuous, the values of the slopes have to approximate the same value. I calculated for checking it. It is 2.
\end{abstract}

Amelie related this approach with the continuity of the function. Her this idea was derived from moving the ring through the curve. As Amelie imagined moving the ring at the beginning of step 6, she could justify her explanation related to the approximating to the point A from the points with smaller x-values and the points with bigger x-values.

In this process, Amelie's developmental progression was revealed based on her mental actions. There were two different layers which the later one depended on the former one.

In the former layer, the student constructed the relation between the secant lines and the tangent line. While she was forming this relation, her mental actions were as follow:

1. Approximating the points on the curve to the specific point on the curve.

2. Rotating the secant line around the specific point. 
These two mental actions supported her in understanding how to be formed the tangent line on the curve. She then needed the quantification of the slope of the tangent line. This quantification process also included the mental actions in detail as follow:

MA1: Approximating the $\mathrm{x}$-values of the points to the $\mathrm{x}$-value of a specific point

MA2: Thinking $y$-values changing depending on $x$-values

MA3: Matching the $x$-values of each points to the $y$-values

MA4: Comparing x-values of points with each other additively

MA5: Comparing y-values of points with each other additively

MA6: Comparing multiplicatively the amount of change in the $x$-values in reference to the specific point with the amount of change in the y-values. (There are two quantities, the amount of change in the $\mathrm{x}$-values $(\Delta \mathrm{x})$ and the amount of change in the $y$-values $(\Delta y))$

MA7: Compare the ratio corresponding to slopes of tangent lines at different points.

Amelie initially considered the points which their x-values were bigger than the $\mathrm{x}$ value of the point $A(1,1)$. She then had similar approaches for the points which their $\mathrm{x}$-values were smaller and completed the quantifying process by comparing the two approximation values.

\subsection{Amelie's Algebraic Representation of the Derivative}

The task also encouraged Amelie to write algebraically the slope of the tangent line. The action of approximating to a specific point on the coordinate system triggered her to call on the concept of limit. While using the limit, she also generalized the slope of the secant lines as the average rate of change. While she was calculating the slopes of the secant lines, she converted her inferences from the graphical approach to the algebraic expression [Excerpt 4].

Excerpt 4 .

Amelie: I am searching the slope of the tangent line, I thus examine the slopes of the secant lines. Because I gradually approximate to the given point, I can use the limit.

Researcher: How do you use the limit?

Amelie: I need find the limit of the slope of secant lines, $\Delta y / \Delta x$.

Researcher: Could you write this statement algebraically?

Amelie: There are the value $f(x+a)$ and the value $f(x)$ for the independent variables. I subtract $x$ from $x+a$ and I would write as 


$$
\frac{f(x+a)-f(x)}{(x+a)-(x)}
$$

Amelie wrote a new mathematical expression $\frac{f(x+a)-f(x)}{(x+a)-(x)}$ as to be equal to $\frac{\Delta y}{\Delta x}$. This expression was more descriptor to see how she thought the average rate of change. She approximated the points to the given point over smaller and smaller intervals. She algebraically wrote this procedure with the limit of $\frac{f(x+a)-f(x)}{(x+a)-(x)}$ as $x+a$ approaches $\mathrm{x}$. She did not mention that the interval would approach to 0. She stated "When I assign values for $\mathrm{x}$, I would find the $\mathrm{y}$-values and the change in the $\mathrm{y}$-values. I then examine the ratio of this change [indicating the change in the $y$-values] over this change [indicating the change in the x-values]. I would find the slope in this way. Then, I approximate this point [indicating any point on the curve] to this point [indicating point A]. Finally, I use the limit for finding the result of this approximation."

\subsection{Global View of Derivative}

Amelia initially worked on the slope of the tangent line at point $(1,1)$ and she found that the value of the slope was 2. This was not indicating her global view for the derivative yet. In the step 9 and step 10, she thought the derivative at different points on the curve in order to construct a global view. She similarly calculated the slope of the tangent lines at different points and matched the value of slope to the $\mathrm{x}$-value of a point [Excerpt 5].

Excerpt 5

Amelie: These values [indicating slopes] are double of these values [indicating the $\mathrm{x}$-values of the points].

Researcher: What can you say else?

Amelie: $x$ transforms to 2x.

Researcher: Okay, think this relation. There is only one slope of the lines at these points. What do you recall mathematically? Are there tangent lines at the all points on the curve and also their slopes?

Amelie: Yes.

Researcher: Do the slopes have only one value?

Amelie: They have one value.

Researcher: When you consider these characteristics, what can you say?

Amelia: This relation is a one-to-one function. 
While she was interpreting the values, she stated that the values of the slopes were double of $\mathrm{x}$-values of the points by matching them. However, she could not relate this relation with the function and could not construct the global view of the derivative. In this step, I asked leading questions and mentioned about the characteristics providing the definition of the function. After all, she could say "This relation is a one-to-one function".

By this task including graphical approach to the derivative, although the student constructed an understanding of the derivative at a point, she had difficulties about thinking the derivative as a function.

\section{Discussion and Conclusion}

In this study, I illustrate a calculus student's mental actions based on the perspective of quantitative reasoning while engaging in the task including the graphical approach to the concept of derivative. Results indicated that using the task involving imagining the situations without technological helps might prompts students in reason about the derivative graphically. this task-based interview also provided me to observe the student's actions during learning process.

Although Amelie algebraically explained how to find the slope of a line, she did not reason about the slope of a tangent line when one point on the tangent line was given. One reason of this obstacle could be derived from Amelie's understanding the slope as a ratio but not a rate. As it can be seen in the Figure 4, she had simultaneous view of algebraic ratio to the geometric ratio (Nagle et al., 2019) but she did not use the idea of slope in calculus meaning yet in this stage. Considering that she could explain the quantity of slope in terms of two points on the line, she did not have the concept of the rate which is a reflectively abstracted constant ratio (Thompson, 1994) and did not have an understanding of it as an object (Nagle et al., 2019). Dubinsky (1991) stated that when properly understood, reflective abstraction appears as a description of the mechanism of the development of intellectual thought (p. 98). In that reason, reflective abstraction of the quantity of ratio is crucial in learning the instantaneous rate of change. In interpreting the derivative as a slope of tangent line, the student's schema about the slope was significant. Although, in this study, I did not examine her understanding of the rate in detail, I observed that Amelie interpreted average rate of change while searching the slope of the tangent line. Students have to associate slope with the steepness of the tangent line of the curve at a point in order to interpret 
derivative graphically (Christensen \& Thompson, 2012). Also, it is important for students to relate and justify the slope's corresponding numerical and physical properties (Nagle et al., 2019). The image in the task representing changing the secant lines as to be the tangent line helped the student to think of the slope of the secant line as a steepness physically and numerically. The idea of steepness pushed the student to form the relationship between the slope of the secant line and the tangent line. However, while Amelie was examining moving of the ring through the parabola, she did not create the secant lines by considering amount of the change between two points on the curve. Therefore, in the step 7, I showed the secant lines formed depending on the movement of the ring. She then interpreted the slopes of the secant lines. This result indicates that Amelie's thinking about the secant line was a static shape thinking which she made inferences based on the secant lines' appearance or shape (Moore \& Thompson, 2015). The movement of ring catalyzed her thinking of the change in the slopes of the lines and approximation to the tangent line. The calculus student's understanding of graphically derivative was consistent with the findings from Asiala et al. (1997) and Zandieh (2000). While these researchers presented more broad actions, I articulated her actions by elaborating them based on the perspective of quantitative reasoning. For example, Asiala et al. (1997) explained, as a first layer, that the students connected two points on a curve to form a secant line and computed the slopes of the secant lines. Differently, this study presented that the student had several actions (MA1-MA6) to reason about the secant lines in the quantification process. In order to interiorize of the actions in point to a single process as the two points on the graph get closer and closer together (Asiala et al., 1997, p.10), the student in this study had the actions of approximating the points on the curve to the specific point on the curve and of rotating the secant lines to form construct the secant lines.

Amelie reasoned about the graphs by the idea of approximation. If she worked on the concept of derivative physically, she might have had more difficulties in the learning process. Physical approach necessarily to have deeply rate of change because it is embedded in this idea (Zandieh, 2000). Since the graphical approach supported the student to have visual understanding of the concept, she could progress on the task. Ellis (2011) has emphasized that visual representations of mathematical relationships help students to make inferences and generalizations. In this regard, presenting the task which the student could imagine the situation by the means of the visual representation prompted the student's mental actions. Also, this process may 
support her in improving the idea of rate of change and slope after the teaching process. This reverse process may detail be researched in the future studies.

When she was first asked to think the slope of tangent line, Amelia related this quantity to the derivative. However, she did not explain its underlying reason because she did not have knowledge about the relation between the secant lines and tangent line. Students generally have procedural understanding of mathematical concepts in the high school teaching process (Ferrini-Mundy \& Graham, 1991). The results related to the student's initial understanding showed that she procedurally learned derivative in the high school. Based on this result, high school mathematics teachers give importance to the quantities of average rate of change and instantaneous rate of change to support their students to have conceptual learning of the derivative.

Student thought that the secant line would look like the tangent line as the ring approximates to the fixed point. Considering that the concept of derivative is explained by the concept of limit, the student's thinking on approximating to a point prompted her to relate this case with the concept of limit. Oerthman (2004) has emphasized that students' spontaneous reasoning about approximation can serve as a productive foundation for limit concept and subsequent development of other major concepts in calculus including the derivative. The results also showed that the student could imagine the instantaneously changing on the distance between the $\mathrm{x}$-values of points on the curve and this mental action supported her to be able to write the derivative algebraically. The approach in the study would help the student to interpret the derivative in a corner point and it can be drawn more tangent lines at a corner point, and he function had no derivative at this point. The task might be meaningful for the students ignoring that the function had no derivative at the corner point and sketching one graph for the function $f^{\prime}$ that was continuous and differentiable at that point (e.g. Borji et al., 2018b).

Besides of these results, I saw that there were some obstacles for having global view of the concept of derivative. The global view of derivative requires strong understanding about the concept of function (Sánchez-Matamoros et al., 2015). In the interview, the student examined the slopes of tangent lines at different points on the curve and matched the dependent variable (slopes) to the independent variable ( $\mathrm{x}$ values of the points) in this process. However, she could not interpret the relationship as a function without support.

This study presents the results from one typical calculus student's mental actions. A research involving students at different level can be done and results can be 
compared with each other. Also, the task can be made more detail and students' learning paths while engaging in the task can be examined through a teaching experiment.

\section{References}

Asiala, M., Cottrill, J., Dubinsky, E., \& Schwingendorf, K. E. (1997). The development of students' graphical understanding of the derivative. The Journal of Mathematical Behavior, 16(4), 399-431. https://doi.org/10.1016/s0732-3123(97)90015-8.

Battista, M. T., \& Clements, D. H. (2000). Mathematics curriculum development as a scientific endeavor. In A. E. Kelly \& R. A. Lesh (Eds.), Handbook of research design in mathematics and science education (pp. 737-76o). Erlbaum. https://doi.org/10.4324/9781410602725.

Borji, V., Alamolhodaei, H., \& Radmehr, F. (2018a). Application of the APOS-ACE theory to improve students' graphical understanding of derivative. EURASIA Journal of Mathematics, Science and Technology Education, 14(7), 2947-2967. https://doi.org/10.29333/ejmste/91451.

Borji, V., Font, V., Alamolhodaei, H., \& Sánchez, A. (2018b). Application of the complementarities of two theories, APOS and OSA, for the analysis of the university students' understanding on the graph of the function and its derivative. EURASIA Journal of Mathematics, Science and Technology Education, 14(6), 2301-2315. https://doi.org/10.29333/ejmste/89514.

Christensen, W. M., \& Thompson, J. R. (2012). Investigating graphical representations of slope and derivative without a physics context. Physical Review Special Topics-Physics Education Research, 8(2), 023101. https://doi.org/10.1103/physrevstper.8.023101.

Common Core State Standards Initiative [CCSSI]. (2010). The common core state standards for mathematics. Washington, DC: National Governors Association Center for Best Practices and Council of Chief State School Officers. Retrieved April 4, 2016 from http://www.corestandards. org/the-standards/mathematics.

Delos Santos, A. G., \& Thomas, M. O. J. (2005). The growth of schematic thinking about derivative. In P. Clarkson, A. Downton, D. Gronn, M. Horne, A. McDonough, R. Pierce, \& A. Roche (Eds.) Building connections: Theory, research and practice. Proceedings of the 28th Mathematics Education Research Group of Australasia Conference Vol. 1 (pp. 377-384). MERGA.

Dubinsky, E. (1991). Constructive aspects of reflective abstraction in advanced mathematics. In Epistemological foundations of mathematical experience (pp. 160-202). Springer. https://doi.org/10.1007/978-1-4612-3178-3_9

Ellis, A. B. (2011). Generalizing-promoting actions: How classroom collaborations can support students' mathematical generalizations. Journal for Research in Mathematics Education, 42(4), 308-345. https://doi.org/10.5951/jresematheduc.42.4.0308.

Ferrini Mundy, J., \& Graham, K. G. (1991). An overview of the calculus curriculum reform effort: Issues for learning, teaching, and curriculum development. The American Mathematical Monthly, 98(7), 627-635. https://doi.org/10.2307/2324931.

Firouzian, S. S. (2013). Students' way of thinking about derivative and its correlation to their ways of solving applied problems. Conference on Research in Undergraduate Mathematics Education. Denver, Colorado.

García, M., Llinares, S., \& Sánchez-Matamoros, G. (2011). Characterizing thematized derivative schema by the underlying emergent structures. International Journal of Science and Mathematics Education, 9(5), 1023-1045. https://doi.org/10.1007/s10763-010-9227-2.

Goldin, G. A. (2000). A scientific perspective on structured, task-based interviews in mathematics education research. In A. E. Kelly \& R. A. Lesh (Eds.), Handbook of research design in mathematics and sciences education (pp. 517-545). Lawrence Erlbaum. https://doi.org/10.4324/9781410602725. 
Habre, S., \& Abboud, M. (2006). Students' conceptual understanding of a function and its derivative in an experimental calculus course. The Journal of Mathematical Behavior, 25(1), 57-72. https://doi.org/10.1016/j.jmathb.2005.11.004.

Hausknecht, A. O., \& Kowalczyk, R. E. (2008). Exploring calculus using innovative technology. In J. Foster (Ed.), Proceedings of the 19th Annual International Conference on Technology in Collegiate Mathematics (pp.75-79). Boston, Massachusetts.

Jones, S. R., \& Watson, K. L. (2018). Recommendations for a "target understanding" of the derivative concept for first-semester calculus teaching and learning. International Journal of Research in Undergraduate Mathematics Education, 4(2), 199-227. https://doi.org/10.1007/s40753-017-0057-2.

Kendal, M., \& Stacey, K. (2000). Acquiring the concept of the derivative: Teaching and learning with multiple representations and CAS. In T. Nakahara \& M. Koyama (Eds.) Proceedings of the 24th Conference of the International Group for the Psychology of Mathematics Education, Vol 3. (pp. 127-134), Hiroshima.

Kertil, M. (2014). Pre-service elementary mathematics teachers' understanding of derivative through a model development unit (Unpublished doctoral dissertation). Middle East Technical University, Ankara, Turkey.

Leinhardt, G., Zaslavsky, O., \& Stein, M. K. (1990). Functions, graphs, and graphing: Tasks, learning, and teaching. Review of Educational Research, 6o(1), 1-64. https://doi.org/10.3102/00346543060001001.

Moore, K. C. (2013). Making sense by measuring arcs: A teaching experiment in angle measure. Educational Studies in Mathematics, 83(2), 225-245. https://doi.org/10.1007/s10649-0129450-6.

Moore, K. C., \& Thompson, P. W. (2015). Shape thinking and students' graphing activity. In Proceedings of the 18th Meeting of the MAA Special Interest Group on Research in Undergraduate Mathematics Education (pp. 782-789). RUME.

Nagle, C., Martínez-Planell, R., \& Moore-Russo, D. (2019). Using APOS theory as a framework for considering slope understanding. The Journal of Mathematical Behavior, 54, 100684. https://doi.org/10.1016/j.jmathb.2018.12.003.

Oehrtman, M. (2004, October). Approximation as a foundation for understanding limit concepts. In Proceedings of the Twenty-Sixth Annual Meeting of the North American Chapter of the International Group for the Psychology of Mathematics Education (Vol. 1, pp. 95-102). Toronto: University of Toronto.

Park, J., Park, M. S., Park, M., Cho, J., \& Lee, K. H. (2013). Mathematical modelling as a facilitator to conceptualization of the derivative and the integral in a spreadsheet environment. Teaching Mathematics and its Applications: An International Journal of the IMA, 32(3), 123-139. https://doi.org/10.1093/teamat/hrto12.

Samuels, J. (2017). A graphical introduction to the derivative. The Mathematics Teacher, 111(1), 4853. https://doi.org/10.5951/mathteacher.111.1.0048.

Sánchez-Matamoros, G., Fernández, C., \& Llinares, S. (2015). Developing pre-service teachers' noticing of students' understanding of the derivative concept. International Journal of Science and Mathematics Education, 13(6), 1305-1329. https://doi.org/10.1007/s10763014-9544-y.

Serhan, D. (2000). The effect of using graphing calculations on students' concept images of the derivative at a point [Doctoral dissertation, Arizona State University]. ProQuest Dissertations and Theses.

Smith III, J. P. J., \& Thompson, P. W. (2007). Quantitative reasoning and the development of algebraic reasoning. In J. J. Kaput, D. W. Carraher, \& M. L. Blanton (Eds.), Algebra in the early grades (pp. 95-132). Erlbaum.

Tall, D. (1997). Functions and calculus. In A. J. Bishop, K. Clements, C. Keitel \& J. Kilpatrick (Eds.), International Handbook of Mathematics Education (pp. 289-325). Kluwer. https://doi.org/10.1007/978-94-009-1465-0. 
Tall, D. (2010). A sensible approach to the calculus (Plenary talk), National and International Meeting on the Teaching of Calculus, Puebla, Mexico. Retrieved from http://homepages.warwick.ac.uk/staff/David.Tall/downloads.html

Thompson, P. W. (1990). A theoretical model of quantity-based reasoning in arithmetic and algebraic. Center for Research in Mathematics \& Science Education: San Diego State University.

Thompson, P. W. (1993). Quantitative reasoning, complexity, and additive structures. Educational studies in Mathematics, 25(3), 165-208. https://doi.org/10.1007/bfo1273861.

Thompson, P. W. (1994). Students, functions, and the undergraduate curriculum. In E. Dubinsky, A. H. Schoenfeld, \& J. J. Kaput (Eds.), Research in Collegiate Mathematics Education, 1 (Issues in Mathematics Education, Vol. 4, pp. 21-44). American Mathematical Society. https://doi.org/10.1090/cbmath/004.

Verhoef, N. C., Coenders, F., Pieters, J. M., van Smaalen, D., \& Tall, D. O. (2015). Professional development through lesson study: Teaching the derivative using GeoGebra. Professional Development in Education, 41(1), 109-126. https://doi.org/10.1080/19415257.2014.886285.

Weber, E. (2013). A learning trajectory for directional derivative. Proceedings of the 35th Annual Meeting of the North American Chapter of the International Group for the Psychology of Mathematics Education (s. 576). Chicago, IL: University of Illinois at Chicago.

Weber, E., Tallman, M., Byerley, C., \& Thompson, P.W. (2012). Derivative as a rate of change. Mathematics Teacher. 106(4), 274-278.

Weber, E., Ellis, A., Kulow, T., \& Ozgur, Z. (2014). Six principles for quantitative reasoning and $\begin{array}{llll}\text { modeling. } & \text { Mathematics } & \text { 24-30. }\end{array}$ https://doi.org/10.5951/mathteacher.108.1.0024.

Weigand, H. G. (2014). A discrete approach to the concept of derivative. ZDM, 46(4), 603-619. https://doi.org/10.1007/s11858-014-0595-X.

Zandieh, M. (2000). A theoretical framework for analyzing student understanding of the concept of derivative. CBMS Issues in Mathematics Education, 8, 103-122. https://doi.org/10.1090/cbmath/008/06. 